\title{
Intramyocardial delivery of bFGF with a biodegradable and thermosensitive hydrogel improves angiogenesis and cardio-protection in infarcted myocardium
}

\author{
HONGLING ZHU ${ }^{1,2}$, XIAOYAN LI ${ }^{1,2}$, MINGJIE YUAN ${ }^{1,2}$, WEIGUO WAN $^{1,2}$, \\ MIAOYANG HU ${ }^{1,2}$, XIAODING WANG ${ }^{1,2}$ and XUEJUN JIANG ${ }^{1,2}$ \\ ${ }^{1}$ Department of Cardiology; ${ }^{2}$ Cardiovascular Research Institute, Renmin Hospital of Wuhan University, \\ Wuhan, Hubei 430060, P.R. China
}

Received April 3, 2016; Accepted March 17, 2017

DOI: 10.3892/etm.2017.5015

\begin{abstract}
Basic fibroblast growth factor (bFGF), a known angiogenic factor, may provide a potential strategy for the treatment of myocardial infarction (MI), but it is limited by a relatively short half-life. Dex-PCL-HEMA/PNIPAAm hydrogel provides a reservoir for the controlled release of growth factors. The aim of the current study was to evaluate the effects of bFGF incorporated into a Dex-PCL-HEMA/PNIPAAm hydrogel on angiogenesis and cardiac health in a rat model of acute MI, induced by coronary artery ligation. Phosphate-buffered solution (PBS group), Dex-PCL-HEMA/PNIPAAm hydrogel (Gel group), bFGF in phosphate-buffered solution (bFGF group) or bFGF in hydrogel (Gel + bFGF group) was injected into a peri-infarcted area of cardiac tissue immediately following MI. On day 30 post-surgery, cardiac function was assessed by echocardiography, apoptosis index by terminal deoxynucleotidyl transferase dUTP nick-end labeling assessment and vascular development by immunohistochemical staining. The findings demonstrated that injection of bFGF along with hydrogel induced angiogenesis, reduced collagen content, MI area and cell apoptosis and improved cardiac function compared with the injection of either bFGF or hydrogel alone. bFGF incorporated with Dex-PCL-HEMA/PNIPAAm hydrogel injection induces angiogenesis, attenuates cardiac remodeling and improves cardiac function following MI.
\end{abstract}

\section{Introduction}

Myocardial infarction (MI) remains one of the most serious cardiovascular diseases worldwide and causes high mortality

Correspondence to: Dr Xuejun Jiang, Department of Cardiology, Renmin Hospital of Wuhan University, 238 Jiefang Road, Wuhan, Hubei 430060, P.R. China

E-mail: xjjiang@whu.edu.cn

Key words: hydrogel, basic fibroblast growth factor, myocardial infarction, angiogenesis and morbidity (1). Acute blocking of the coronary artery leads to acute ischemia, hypoxia and irreversible death in myocardial cells, eventually inducing adverse cardiac remodeling and progressive heart failure (2-4). Thus, in situ repair and regeneration of vessels to rebuild coronary collateral circulation and improve blood supply to the infarction zone and its periphery have emerged as potential therapeutic approaches for treating MI $(5,6)$.

Growth factors are widely used to treat MI by inducing angiogenesis (7). Fibroblast growth factors (FGFs) are potent mitogens that induce angiogenesis in vascular and capillary endothelial cells. Basic FGF (bFGF), a 16-kD monomeric factor, is the most potent angiogenic factor in the FGF family, and it targets the migration and proliferation of endothelial cells, smooth muscle cells and fibroblasts $(8,9)$. bFGF has been extensively studied in animal $(10-12)$ and clinical $(13,14)$ experiments.

However, as bFGF has a short half-life and because the improper administration of bFGF at high doses is accompanied by side effects, including retinopathy $(15,16)$, hypotension $(17)$, angioma, and edema and thrombocytopenia $(8,9)$, the therapeutic administration of bFGF is largely limited to laboratory and clinical trials.

Thermosensitive Dex-PCL-HEMA/PNIPAAm hydrogel is a type of cross-linked polymer with biocompatibility and nontoxicity (18). In a previous study, hydrogel was identified to be a suitable delivery vehicle and controlled-release system for drugs and cells. He et al (19) combined this hydrogel with high mobility group box 1 and injected it into the peri-infarct zone of a rat heart, which induced the activation of endogenous c-Kit cardiac cells and vascular regeneration. Wan et al (20) combined this hydrogel with short hairpin RNA of angiotensin converting enzyme, which induced enhanced cardioprotective effects in a rat with MI.

Thus, the aim of the present study was to investigate whether the angiogenesis effect induced by bFGF is strengthened or enhanced by incorporating it into a hydrogel (a controlled delivery system) to sustain and localize release in the infarcted heart wall of a rat model of MI, and also to investigate whether bFGF and hydrogel implantation may improve infarcted left ventricle (LV) function, and inhibit LV remodeling. 


\section{Materials and methods}

Animals. A total of 52 8-10 week old male Sprague Dawley rats (200-250 g) were used. Rats were purchased from Hunan Provincial Center for Disease Control and Prevention (Beijing, China) and maintained in a controlled environment with temperature at $22-25^{\circ} \mathrm{C}$, humidity at $45-55 \%$, and a $12: 12-\mathrm{h}$ light-dark cycle. Groups of five rats were housed in a cage with food and water provided ad libitum. All experimental procedures involving animal use conformed with the Guide for the Care and Use of Laboratory Animals, as published by the US National Institutes of Health, and the protocol was approved by the Institutional Animal Care Committee from Wuhan University (Wuhan, China).

Synthesis of hydrogel. All the reagents used to synthesize the hydrogel, including N-isopropylacrylamide (NIPAAm), 2-Hydroxylethyl methacrylate (HEMA) and dextran were obtained from Shanghai Chemical Reagent Co., Ltd. (Shanghai, China). As described previously (18), the hydrogel was synthesized with biodegradable dextran chains grafted with a hydrophobic poly (e-caprolactone)-HEMA (PCL-HEMA) chain and the PCL (polycaprolactam)-grafted polysaccharide chains into a thermoresponsive poly-NIPAAm (PNIPAAm) network. The former is hydrophobic and the latter is amphiphilic and thermoresponsive. Thus, the hydrogel is biodegradable, biocompatible and thermoresponsive. The gel solution has a lower critical solution temperature (LCST) of $33^{\circ} \mathrm{C}$ and is therefore able to shift from solution to gel at $37^{\circ} \mathrm{C}$ and solidify at a temperature below the LCST. The polymer was mixed with phosphate-buffered solution (PBS) to develop the $1.5 \%$ weight (WT) gel solution and was subsequently autoclaved at $120^{\circ} \mathrm{C}$ for $20 \mathrm{~min}$ for sterilization.

In vitro release of bFGF from hydrogel. Initially, 100 units of bFGF was dissolved in $1.0 \mathrm{ml}$ Dex-PCL-HEMA/PNIPAAm hydrogel solution $(1.5 \% \mathrm{WT})$, and air bubbles were prevented during the mixing process. The mixture was then combined with $500 \mathrm{ml} \mathrm{PBS}$ in a beaker at $37^{\circ} \mathrm{C}$. At 1, 5, 10, 15, 20, 25 and 30 days after the combination during the in vitro release experiment, a $1 \mathrm{ml}$ aliquot of the buffer medium was removed for bFGF concentration measurement. The concentration of bFGF released from the hydrogel was analyzed using a human bFGF chemiluminescence immunoassay diagnostic kit (xy-CL-H0394c; Siemens AG, Munich, Germany); and a chemiluminescence immunoassay analyzer machine (IMMULITE $^{\circledR}$ 2000; Siemens AG) according to the manufacturer's protocol. Dex-PCL-HEMA/PNIPAAm hydrogel without bFGF was used as a control. Cumulative bFGF release (\%) from the hydrogel was calculated as follows: Cumulative release $(\%)=(\mathrm{Mt} / \mathrm{M} 0) \times 100$, where $\mathrm{Mt}$ is the amount of bFGF released from the hydrogel at time $(\mathrm{t})$ and $\mathrm{M} 0$ is the concentration of $100 \mathrm{U}$ of bFGF dissolved in $500 \mathrm{ml}$ PBS. Assessments of the bFGF release from the hydrogel were repeated three times.

Model of MI and in vivo bFGF delivery. All 52 male Sprague Dawley rats were anesthetized with 3\% sodium pentobarbital (p3761; Sigma-Aldrich; Merck KGaA, Darmstadt, Germany) $(30 \mathrm{mg} / \mathrm{kg})$ peritoneally, intubated and ventilated at a respiratory rate of 70 times/min. A thoracotomy was then performed at the third left intercostal space. Following stripping of the epicardia and identification of the anatomy, the left coronary artery was ligated with 6-to-0 polypropylene stitching, $2 \mathrm{~mm}$ below the left atrial appendage. Electrocardiogram (ECG) monitoring was used and when the ECG read an ST-segment elevation in Leads II and III and pallor appeared in the infarcted area, this indicated successful MI. Rats with MI were randomly assigned to groups receiving multiple intramyocardial injections of $100 \mu \mathrm{l}$ PBS without bFGF (group PBS), $100 \mu 1$ hydrogel without bFGF (group Gel), $100 \mu 1$ PBS containing $2 \mu \mathrm{g}$ bFGF (PBS + bFGF; group bFGF) or $100 \mu \mathrm{l}$ hydrogel containing $2 \mu \mathrm{g}$ bFGF (hydrogel + bFGF; group Gel + bFGF). Each group had 7-10 rats available for testing. The compound was injected via a 32-gauge needle into four sites ( $25 \mu \mathrm{l}$ per injection) of the peri-infarct zone immediately following coronary ligation.

Echocardiography. On day 30 following MI, all of the living rats were anesthetized with $3 \%$ sodium pentobarbital $(25 \mathrm{mg} / \mathrm{kg}$ ) injected peritoneally and placed in a lateral position, with their chest shaved. In a blind manner, transthoracic echocardiography (Acuson; Siemens Healthcare, Mountain View, CA, USA) equipped with a 3- to 7-MHz probe was used to assess their left ventricular (LV) dimension and function through long-axis and short-axis views. Rats with a visual infarct area $<20 \%$ at the papillary level in the short-axis view were excluded to minimize the variation in infarct areas. LV end-diastolic diameter (LVEDD), LV end-systolic diameter (LVESD) and LV ejection fraction (LVEF) were measured from at least three consecutive cardiac cycles.

Histopathological examinations. Following the echocardiography measurements on day 30 post-MI, the rats were sacrificed by sodium pentobarbital overdose $(200 \mathrm{mg} / \mathrm{kg})$. The heart was quickly removed in diastole with $10 \% \mathrm{KCl}$. Ventricles were fixed in $10 \%$ ( $\mathrm{vol} / \mathrm{vol}$ ) buffered formalin solution at $4^{\circ} \mathrm{C}$ for $24-48 \mathrm{~h}$. After dehydration with a graded series $[75,85,90,95$ and $100 \%$ (vol/vol)] of ethanol solution, five different transverse levels around the injection site were subsequently embedded. The specimens were sliced into 5- $\mu \mathrm{m}$ thick sections from apex to base and stained with Masson trichrome and underwent immunohistochemistry analysis.

Firstly, the sections were rinsed in peroxidase blocking agent (K4011; Dako; Agilent Technologies, Inc., Santa Clara, CA, USA) at room temperature for 5 min to block endogenous peroxidase activity, then washed with PBS 3 times (5 $\mathrm{min} /$ wash). Secondly, the sections were incubated with the following primary antibodies at $4^{\circ} \mathrm{C}$ overnight to analyze neovascularization: Anti- $\alpha$-smooth muscle actin ( $\alpha$ SMA) antibody (MABT381; 1:100 dilution; EMD Millipore, Billerica, MA, USA) and anti-cluster of differentiation (CD)31 antibody (sc-1506; 1:100 dilution; Santa Cruz Biotechnology, Inc., Dallas, TX, USA). Sections were then washed with PBS. Thirdly, horseradish peroxidase (HRP)-conjugated goat anti-rabbit antibody (K4011; Dako; Agilent Technologies, Inc.) and HRP-conjugated rabbit anti-goat antibody (14-13-06, KPL, Inc., Gaithersburg, MD, USA) were used as secondary antibodies and the incubation extended for 30-60 min at room temperature. Finally, sections were rinsed in diaminobenzidine substrate-chromogen solution (K4011, Dako; Agilent 
Technologies, Inc.) for $10 \mathrm{~min}$ and subsequently in hematoxylin solution (03971; Sigma-Aldrich; Merck KGaA) for a further $10 \mathrm{sec}$ for counterstaining. Sections were washed with PBS and prepared for testing.

The MI area, collagen content and new blood vessels were examined via light microscopy in sections taken from the border zone (1-2 $\mathrm{mm}$ from the edge of the infarction zone) of the four groups. In each LV transverse section, four random microscopic fields were selected in a blind fashion and subsequently examined. The infarct size (\%) was calculated from the ratio of the surface area of the infarct wall and the entire surface area of the left ventricle by automated computer image analysis. The collagen content (\%) was calculated from the ratio of the collagen area to the area of the entire high-powered field (magnification, x200) using image analysis software (Image-Pro Plus 6.0; Media Cybernetics, Inc, Rockville, MD, USA). The number of arterioles/high-powered field (magnification, x200) and capillaries/high-powered field (magnification, $\mathrm{x} 200$ ) were counted in three tissue sections from each rat. The mean results of data compiled from the four fields of each section were subsequently calculated. Blood vessel density was indicated as the number of vessels at one high-powered field (magnification, x200).

TerminaldeoxynucleotidyltransferasedUTPnick-endlabeling (TUNEL) assay. On day 30, a TUNEL assay (11684817910; Roche Diagnostics GmbH, Mannheim, Germany) was used to detect apoptotic cardiac cells in the LV. As directed by the manufacturer, this method uses the transferase-mediated dUTP nick-end labeling technique to stain DNA fragments in the nucleus of apoptotic cells, followed by hematoxylin counterstaining. A total of three different fields (magnification, $\mathrm{x} 200$ ) from each slide were examined by light microscopy. TUNEL-positive nuclei and the total number of nuclei were recorded by a blinded rater. The percentage of TUNEL positive nuclei was deemed as the apoptotic index.

Statistical analysis. Data are presented as the mean \pm standard deviation. Statistical analysis of the data were performed by one-way analysis of variance followed by the Student-Newman-Keuls' test. $\mathrm{P}<0.05$ was considered to indicate a statistically significant difference.

\section{Results}

Animals. A total of 52 rats were initially included in the present study. Prior to surgery, 2 rats succumbed to hyperaesthesia, 5 (1 in PBS, 1 in Gel, 1 in Gel + bFGF and 2 in bFGF group) succumbed to acute left heart failure within $24 \mathrm{~h}$ of MI surgery and another 2 (1 in PBS and 1 in Gel group) due to wound infection. A total of 3 rats (1 in PBS, 1 in Gel and 1 in Gel + bFGF group) were excluded because the infarct size was $<20 \%$ on visual inspection. From each group, 30 days post-MI after echocardiography testing, 7 rats were sacrificed to measure the infarct size, collagen content and angiogeneisis, in addition to a TUNEL assay. Echocardiography was performed in all the rats, 30 days post MI.

In vitro release kinetics of bFGF from the hydrogel. In vitro release rates of bFGF from the hydrogel are presented in
Fig. 1. Within 5 days, $\sim 60 \%$ of the bFGF was released from the hydrogel and, by day $10, \sim 72 \%$ of the bFGF was released. By day 30, $>95 \%$ was released.

$L V$ diameter and LVEF. On day 30 post-MI, the Gel, bFGF and $\mathrm{Gel}+\mathrm{bFGF}$ groups exhibited a significant reduction in $\mathrm{LV}$ diameter $(\mathrm{P}<0.05$; Fig. $2 \mathrm{~A}$ and $\mathrm{B})$ and a significant increase in the LVEF ( $\mathrm{P}<0.05$; Fig. 2C), compared with the PBS group. The Gel + bFGF group demonstrated a significant decrease in LVEDD and LVESD and a significant increase in LVEF compared with the Hydrogel and bFGF group $(\mathrm{P}<0.05$; Fig. $2 \mathrm{~A}$ and B). Differences in the LV diameters or LVEF between the hydrogel and bFGF groups were not significant.

Infarct size. The Gel, bFGF and Gel + bFGF groups exhibited a significant reduction in infarct size compared with the PBS group on day 30 following $\mathrm{MI}(\mathrm{P}<0.05$; Fig. 3), whereas the Gel + bFGF group demonstrated the largest reduction compared with groups treated with either agent alone. The bFGF group demonstrated a slight reduction in infarct size compared with that of the Gel group, but it was not significant.

Collagen content. On day 30 post-MI, the collagen content was significantly decreased following treatment with bFGF and hydrogel compared with the results of using either agent alone ( $\mathrm{P}<0.05$; Fig. 4). Although the PBS, Gel and bFGF groups indicated no significant differences in collagen content, the bFGF group exhibited a slightly decreased collagen content compared with that of the Gel and PBS group.

Apoptosis. The bFGF group and the Gel + bFGF group exhibited a significant decrease $(\mathrm{P}<0.05$; Fig. 5$)$ in the apoptosis index when compared with the PBS and Gel groups 30 days after MI, whereas the Gel + bFGF group had a significantly reduced apoptosis index $(\mathrm{P}<0.05$; Fig. 5) compared with the bFGF group. There was no significant difference in the apoptotic index between the PBS group and the Gel group (Fig. 5).

Neovascularization: $\alpha-S M A$ and CD31. On day 30 post-MI, the arterial density and capillary density in the peri-infarct area was significantly increased in the bFGF group and the $\mathrm{Gel}+\mathrm{bFGF}$ group compared with the other groups $(\mathrm{P}<0.05$; Figs. 6 and 7), whereas the Gel + bFGF group exhibited a significant increase in arterioles, compared with the bFGF group $(\mathrm{P}<0.05$; Figs. 6 and 7$)$. No significant difference in arteriole density was observed between the PBS group and the Gel group.

\section{Discussion}

The present study is, to the best of our knowledge, the first to demonstrate that an intramyocardial injection of bFGF along with a Dex-PCL-HEMA/PNIPAAm hydrogel immediately following $\mathrm{MI}$ in rats significantly decreases the size of the infarct zone for up to 30 days, inhibits the apoptosis of cardiomyocytes, increases angiogenesis, attenuates ventricular remodeling, and improves cardiac function compared with injection of either alone or PBS treatment.

Angiogenic therapy is emerging as a potential strategy for the treatment of ischemic heart disease. As growth factors have 


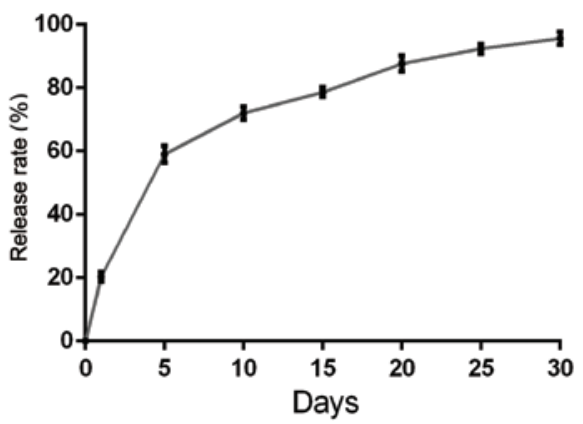

Figure 1. Time course of the in vitro basic fibroblast growth factor release from the Dex-PCL-HEMA/PNIPAAm hydrogel over 30 days.
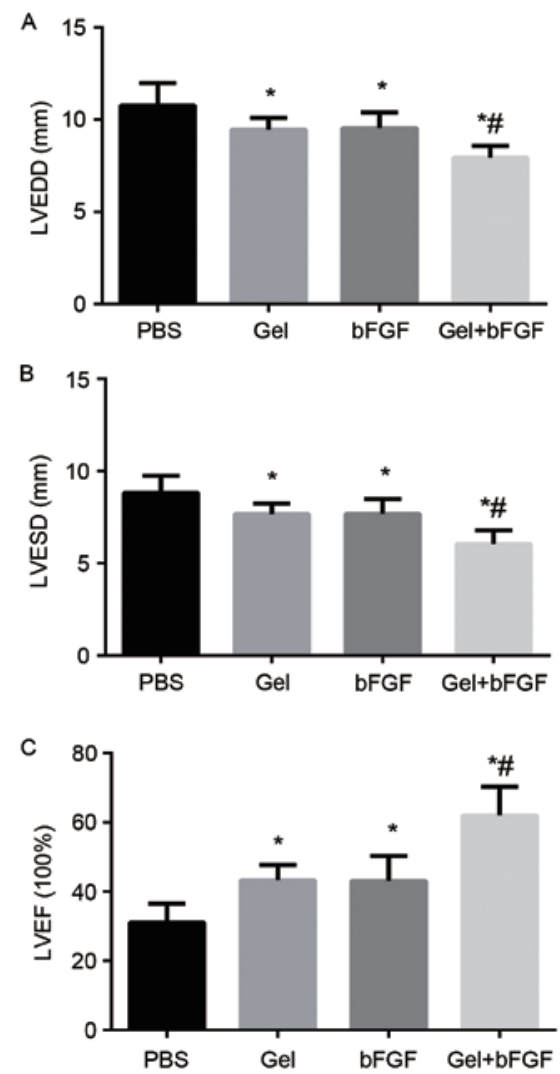

Figure 2. Echocardiographic evaluation of LV function. Evaluation of (A) LVEDD, (B) LVESD and (C) LVEF 30 days post-MI. Data are expressed as the mean + standard deviation, ${ }^{\text {"P }}<0.05$ vs. $\mathrm{PBS}$ and Gel; ${ }^{\text {"P }} \mathrm{P}<0.05$ vs. bFGF ( $\mathrm{n}=7$ in each group). LV, left ventricular; LVEDD, LV end-diastolic diameter; LVESD, LV end-systolic diameter; LVEF, LV ejection fraction MI, myocardial infarction; bFGF, basic fibroblast growth factor; PBS, phosphate buffered saline; Gel, rats treated with $100 \mu 1$ hydrogel without bFGF; Gel + bFGF, rats treated with $100 \mu 1$ PBS containing $2 \mu \mathrm{g} \mathrm{bFGF}$.

a short half-life, numerous approaches have been used to deliver them in vivo, including intracoronary delivery, intravenous delivery and left atrial administration. $(8,9,21,22)$ However, these methods cannot ensure an adequate concentration of the growth factors in the targeted myocardium as general blood circulation accelerates their metabolism and degradation (23). Lazarous et al (24) demonstrated that when $\sim 100 \mu \mathrm{g} / \mathrm{kg}$ bFGF was injected per animal, only $3-5 \%$ of the total dose was recovered from the heart following intracoronary administration, $1.3 \%$ following left atrial administration, $0.5 \%$ following

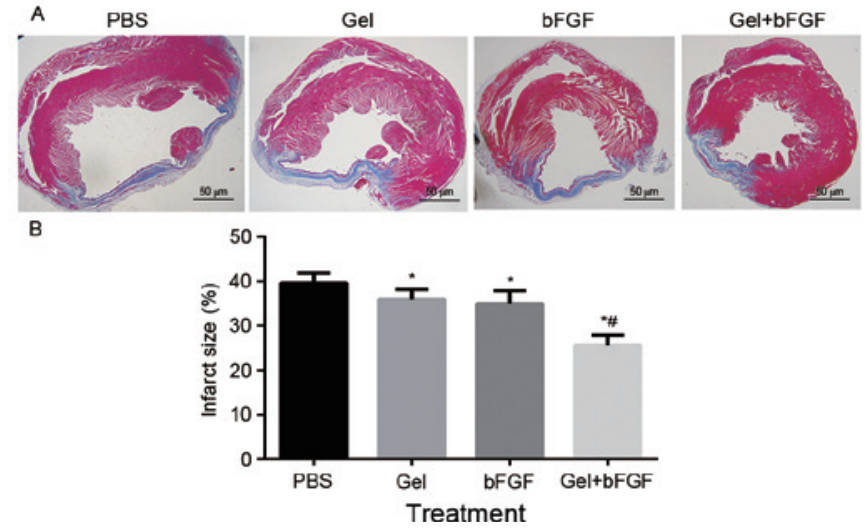

Figure 3. Infarct size on day 30 post-MI. (A) Representative images of left ventricles from each group following Masson's Trichrome staining (magnification, $\mathrm{x} 10$ ). (B) Infarct size as percentages at 30 days. Infarct size is calculated from the ratio of surface area of infarct wall and the entire surface area of the left ventricle. Data are expressed as the mean + standard deviation, ${ }^{~} \mathrm{P}<0.05$ vs. $\mathrm{PBS}$ and $\mathrm{Gel} ;{ }^{*} \mathrm{P}<0.05$ vs. $\mathrm{bFGF}$ ( $\mathrm{n}=7$ in each group). MI, myocardial infarction; PBS, phosphate buffered saline; Gel, rats treated with $100 \mu 1$ hydrogel without bFGF; bFGF, basic fibroblast growth factor; Gel + bFGF, rats treated with $100 \mu 1$ PBS containing $2 \mu \mathrm{g}$ bFGF.

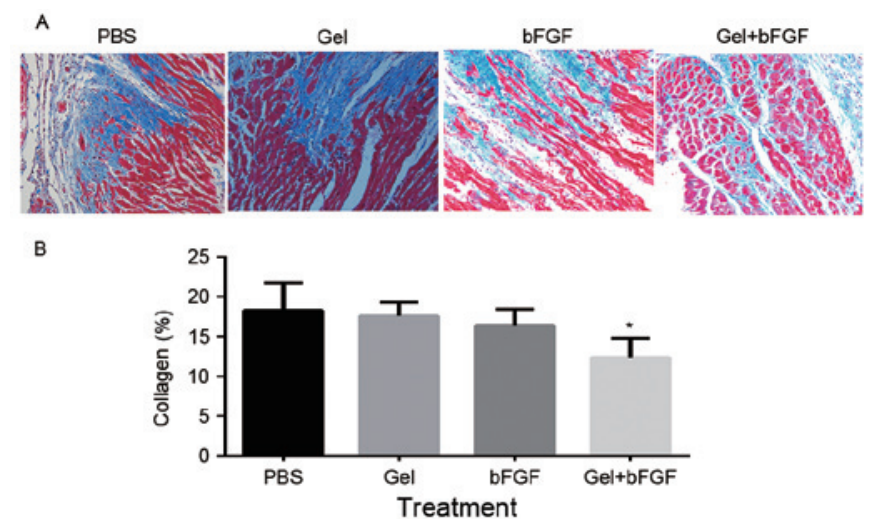

Figure 4. Collagen content 30 days post-MI. (A) Representative images of left ventricles from each group following Masson's Trichrome staining (magnification, x200). (B) Collagen content as percentages on day 30. Collagen content is calculated from the ratio of the collagen area to the area of the entire high-power field. Data are expressed as the mean + standard deviation, ${ }^{*} \mathrm{P}<0.05$ vs. PBS and Gel ( $\mathrm{n}=7$ in each group). MI, myocardial infarction; PBS, phosphate buffered saline; Gel, rats treated with $100 \mu 1$ hydrogel without bFGF; bFGF, basic fibroblast growth factor; Gel + bFGF, rats treated with $100 \mu \mathrm{l}$ PBS containing $2 \mu \mathrm{g}$ bFGF.

intravenous or Swanz Ganz delivery, and 19\% $150 \mathrm{~min}$ after pericardial administration. Furthermore, such delivery methods with a high dose of bFGF have side effects, including proatherogenic effects, plaque expansion and instability, rheumatoid arthritis, hemangiomas and atherosclerosis (25). An intramyocardial route provides a high myocardial uptake of angiogenesis factors. Kawasuji et al (26) demonstrated that intramyocardial administration of bFGF increased regional myocardial blood flow and improved cardiac function in acute MI, indicating that intramyocardial administration of bFGF may be a possible therapeutic approach for patients with acute MI. Similarly, in the current study when compared with the PBS group, the bFGF group presented with a smaller infarct size, less collagen deposition, a smaller apoptosis index, more angiogenesis and more preserved cardiac function. 


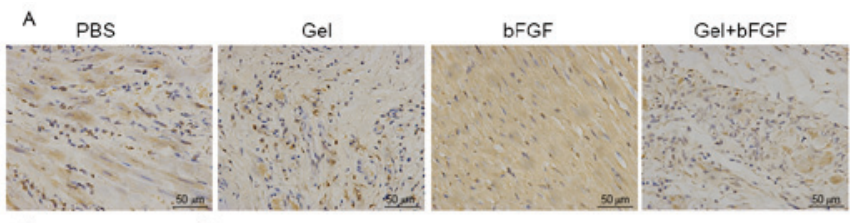

B

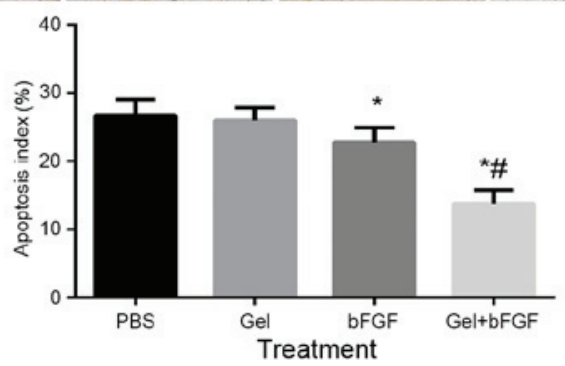

Figure 5. Cardiomyocyte apoptosis 30 days post-MI.(A) Immunohistochemistry of terminal deoxynucleotidyl transferase dUTP nick-end labeling in the border zone (magnification, x200). (B) Apoptotic index was quantified at 30 days. Data are expressed as the mean \pm standard deviation, ${ }^{*} \mathrm{P}<0.05$ vs. $\mathrm{PBS}$ and Gel; ${ }^{\text {}} \mathrm{P}<0.05$ vs. bFGF ( $\mathrm{n}=7$ in each group). MI, myocardial infarction; PBS, phosphate buffered saline; Gel, rats treated with $100 \mu 1$ hydrogel without bFGF; bFGF, basic fibroblast growth factor; Gel + bFGF, rats treated with $100 \mu \mathrm{l}$ PBS containing $2 \mu \mathrm{g}$ bFGF.

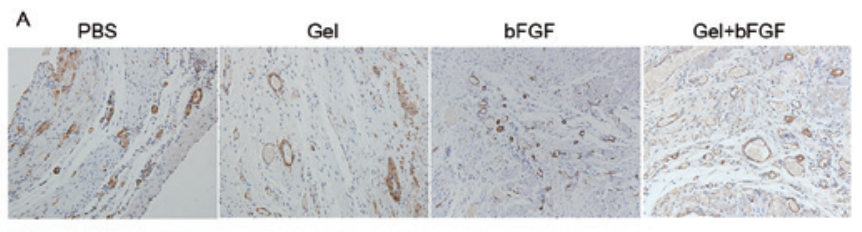

B

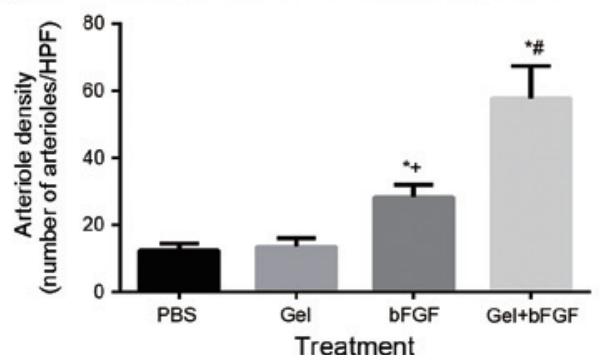

Figure 6. Arterial density 30 days post-MI. (A) Representative immunohistochemistry images of $\alpha$-SMA staining in the border zone (magnification, x200). (B) Arterial density per HPF (magnification, x200) at 30 days. Data are expressed as the mean \pm standard deviation, ${ }^{*} \mathrm{P}<0.05$ vs. $\mathrm{PBS} ;{ }^{+} \mathrm{P}<0.05$ vs. Gel; " $\mathrm{P}<0.05$ vs. bFGF ( $\mathrm{n}=7$ in each group). MI, myocardial infarction; $\alpha$-SMA, $\alpha$-smooth muscle actin; PBS, phosphate buffered saline Gel, rats treated with $100 \mu 1$ hydrogel without bFGF; bFGF, basic fibroblast growth factor; Gel + bFGF, rats treated with $100 \mu 1$ PBS containing $2 \mu \mathrm{g}$ bFGF; HPF, high power field.

Furthermore, the dose of bFGF in the current study was only $8 \mu \mathrm{g} / \mathrm{kg}$ per rat, which was much less than the doses used in previous studies.

Biomaterials including collagen (27), fibrin glue (28), alginate (29), matrigel and self-assembling peptides (30) and hydrogel (31) are widely used in tissue engineering and they are more likely to be used as a delivery and sustained release system for growth factors to induce enhanced myocardial protective effects. Garbern et al (32) used a pH-responsive hydrogel carrying bFGF to induce angiogenesis and achieve therapeutic effects on regional blood flow and cardiac function in infarcted myocardium. However, the dual ability of this hydrogel to respond to both $\mathrm{pH}$ and temperature makes

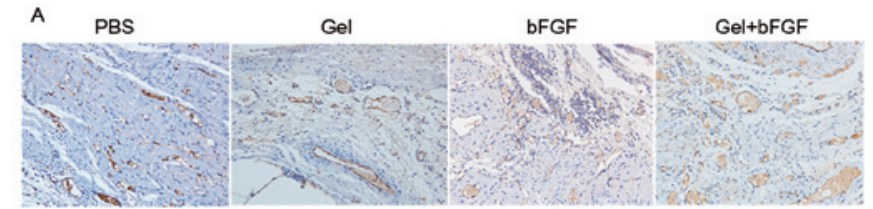

B

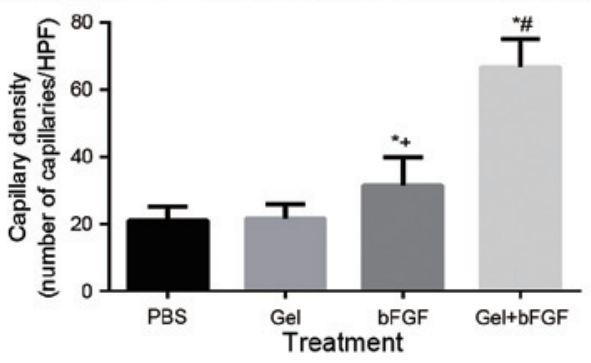

Figure 7. Capillary density 30 days post-MI. (A) Representative images of Immunohistochemistry of CD31 staining in the border zone (magnification, x200). (B) Capillary density per HPF (magnification, x200) at 30 days. Data are expressed as the mean \pm standard deviation, ${ }^{*} \mathrm{P}<0.05$ vs. $\mathrm{PBS} ;{ }^{+} \mathrm{P}<0.05$ vs. $\mathrm{Gel}$; ${ }^{\mathrm{P}}<0.05$ vs. bFGF ( $\mathrm{n}=7$ in each group). MI, myocardial infarction; CD31, cluster of differentiation 31; PBS, phosphate buffered saline; Gel, rats treated with $100 \mu 1$ hydrogel without bFGF; bFGF, basic fibroblast growth factor; Gel + bFGF, rats treated with $100 \mu$ l PBS containing $2 \mu \mathrm{g}$ bFGF; HPF, high power field.

it uncontrollable in the presence of an infarcted myocardium with a pathological microenvironment. The sustained release of bFGF with an alginate microsphere induced a valid improvement in treating MI but the alginate is a polysaccharide with poor biodegradability (33). A combination of polyvinyl alcohol-dextran blended hydrogel with bFGF stimulated angiogenesis and increased wall thickness in an infarcted myocardium but did not increase cardiac function 2 months after MI (34). In a study by Nie et al (7), bFGF integrated with a fibrin glue in a canine infarct model improved myocardial perfusion and cardiac function, but the fibrin interacted with cells from the blood and vessel walls, which would inevitably impair the bindings of bFGF to relevant receptors (7).

Compared with these materials, the hydrogel used in the current study has a number of advantages. The Dex-PCL-HEMA/PNIPAAm hydrogel is biodegradable, biocompatible and nontoxic. Furthermore, this hydrogel is thermosensitive and exhibits a $\operatorname{LCST}\left(\sim 33^{\circ} \mathrm{C}\right)$ and solidifies at $37^{\circ} \mathrm{C}(18,35)$. Thus, in vitro the liquid hydrogel uniformly mixed with bFGF and, when it was injected into the heart tissue, the hydrogel solidified instantly in situ at body temperature. The rapid gelation kinetics of the hydrogel guaranteed the effective entrapment of biologically active additives at the injection site. The solid hydrogel serves as a controlled release system and caused local and sustained release of bFGF at a relatively high concentration. As the release kinetics of bFGF from the hydrogel in vitro demonstrated, even 30 days following mixing, bFGF was still being released from the hydrogel.

Furthermore, the hydrogels are cross-linked and porous polymer networks that may absorb a large amount of water and exchange oxygen, nutrients and other metabolites, thus making hydrogels similar to the cardiac extracellular matrix (ECM). The hydrogelism ECM may replace certain damaged ECM functions and provide a suitable microenvironment for the infarcted myocardiums, which results in a smaller infarct 
size and less collagen deposition. The solid hydrogel also has a controllable mechanical strength, which may provide structural and mechanical support for the infarcted wall and prevent paradoxical motion at the infarct site.

As a previous study demonstrated, a MI of the Dex-PCL-HEMA/PNIPAAm hydrogel may significantly inhibit infarct ventricle remodeling and improve cardiac function by thickening and toughening the infarcted wall and reducing paradoxical motion (35). Therefore, this hydrogel serves as an effective controlled release system to deliver proteins, genes and cells at the infarct site in a sustained and local manner and to induce enhanced cardiac protective effects compared with using either hydrogel or bioactive additives alone $(19,20,36)$. For example, a MI of a hydrogel combined with vascular endothelial growth factor-165 in the rat heart 30 days following MI stimulation has been demonstrated to enhance angiogenesis and improve cardiac function (37).

Similarly, the present study indicated that the delivery of bFGF with a Dex-PCL-HEMA/PNIPAAm hydrogel may also improve angiogenesis and cardioprotection in infarcted myocardium. In the bFGF + Gel group, the biocompatibility and thermosensitivity of the hydrogel served as a controlled release system and caused bFGF to accumulate at relatively high concentrations in the peri-infarct areas. It induced more angiogenesis than that of the bFGF group. Furthermore, the porous polymer physical structure of the hydrogel allows the absorbion of a large amount of water and nutrients, and it may serve as a supporting wall at the peri-infarct site. The bFGF + Gel group also had a smaller infarct size, less collagen deposition and a smaller apoptosis index. However, in the Gel and bFGF groups, the supporting function of the hydrogel and the angiogenesis activity of the bFGF are less effective than in the combined bFGF + Gel group. Thus, the hydrogel may prolong the release of bFGF and increase the local concentration of bFGF by protecting against extracellular degradation. The inherent function of the hydrogel itself may be the mechanism by which, an intramyocardial injection of hydrogel with bFGF into the peri-infarcted hearts of rats has a more synergistic effect than using bFGF or hydrogel either alone.

A limitation of the present study is that the release kinetics of sustained bFGF delivery from the hydrogel in vivo was not analyzed. Future studies should investigate this and assess different concentrations of bFGF to determine the optimal dosage.

In conclusion, the present study is, to the best of our knowledge, the first to demonstrate that a combination of bFGF and Dex-PCL-HEMA/PNIPAAm hydrogel has synergistic benefits that are superior to the administration of either treatment alone. This combination is able to induce angiogenesis, attenuate cardiac remodeling and improve cardiac function in rats with MI.

\section{Acknowledgements}

The present study was supported by grants from the National Nature Science Foundation of China (grant no. 81170307), National Key Basic Research Program of China (grant no. 2005CB623903) and The Fundamental Research Funds for the Central Universities (grant no. 2042014kf0130). The authors would also like to thank The Nature Publishing Group Language Editing company for editing the original manuscript.

\section{References}

1. Zhang BH, Guo CX, Wang HX, Lu LQ, Wang YJ, Zhang LK, Du FH and Zeng XJ: Cardioprotective effects of adipokine apelin on myocardial infarction. Heart Vessels 29: 679-689, 2014.

2. Mann DL: Mechanisms and models in heart failure: A combinatorial approach. Circulation 100: 999-1008, 1999.

3. Gierach J, Gierach M, Świątkiewicz I, Woźnicki M, Grześk G, Sukiennik A, Koziñski M and Kubica J: Admission glucose and left ventricular systolic function in non-diabetic patients with acute myocardial infarction. Heart Vessels 31: 298-307, 2016.

4. Cleland JG, Torabi A and Khan NK: Epidemiology and management of heart failure and left ventricular systolic dysfunction in the aftermath of a myocardial infarction. Heart 91 (Suppl 2): ii7-13; ii31, ii43-48, 2005.

5. Jośko J, Gwóźdź B, Jedrzejowska-Szypułka H and Hendryk S: Vascular endothelial growth factor (VEGF) and its effect on angiogenesis. Med Sci Monit 6: 1047-1052, 2000.

6. Carmeliet P and Jain RK: Angiogenesis in cancer and other diseases. Nature 407: 249-257, 2000

7. Nie SP, Wang X, Qiao SB, Zeng QT, Jiang JQ, Liu XQ, Zhu XM, Cao GX and Ma CS: Improved myocardial perfusion and cardiac function by controlled-release basic fibroblast growth factor using fibrin glue in a canine infarct model. J Zhejiang Univ Sci B 11: 895-904, 2010

8. Lazarous DF, Shou M, Scheinowitz M, Hodge E, Thirumurti V, Kitsiou AN, Stiber JA, Lobo AD, Hunsberger S, Guetta E, et al: Comparative effects of basic fibroblast growth factor and vascular endothelial growth factor on coronary collateral development and the arterial response to injury. Circulation 94: 1074-1082, 1996.

9. Lazarous DF, Scheinowitz M, Shou M, Hodge E, Rajanayagam S, Hunsberger S, Robison WG Jr, Stiber JA, Correa R, Epstein SE, et al: Effects of chronic systemic administration of basic fibroblast growth factor on collateral development in the canine heart. Circulation 91: 145-153, 1995.

10. Si HB, Zeng Y, Lu YR, Cheng JQ and Shen B: Control-released basic fibroblast growth factor-loaded poly-lactic-co-glycolic acid microspheres promote sciatic nerve regeneration in rats. Exp Ther Med 13: 429-436, 2017.

11. Lin Z, Hu Y, Wang Z, Pan S, Zhang H, Ye L, Zhang H, Fang M, Jiang $\mathrm{H}$, Ye J, et al: Intranasal basic fibroblast growth factor attenuates endoplasmic reticulum stress and brain injury in neonatal hypoxic-ischaemic injury. Am J Transl Res 9: 275-288, 2017.

12. Ran F, Liu C, Liu Z, Shang T, Zhou M and Qiao T: Preventive effects of basic fibroblast growth factor on vascular restenosis after balloon angioplasty. Exp Ther Med 7: 1193-1196, 2014.

13. Kanazawa T, Kurakami K, Kashima K, Konomi U, Komazawa D, Nakamura K, Matsushima K, Akagi Y, Misawa K, Nishino H and Watanabe Y: Injection of basic fibroblast growth factor for unilateral vocal cord paralysis. Acta Otolaryngol 137: 962-967, 2017.

14. Kumagai M, Marui A, Tabata Y, Takeda T, Yamamoto M, Yonezawa A, Tanaka S, Yanagi S, Ito-Ihara T, Ikeda T, et al: Safety and efficacy of sustained release of basic fibroblast growth factor using gelatin hydrogel in patients with critical limb ischemia. Heart Vessels 31: 713-721, 2016.

15. Floege J, Kriz W, Schulze M, Susani M, Kerjaschki D, Mooney A, Couser WG and Koch KM: Basic fibroblast growth factor augments podocyte injury and induces glomerulosclerosis in rats with experimental membranous nephropathy. J Clin Invest 96: 2809-2819, 1995 .

16. Sasaki T, Jyo Y, Tanda N, Kawakami Y, Nohno T, Tamai H and Osawa G: Changes in glomerular epithelial cells induced by FGF2 and FGF2 neutralizing antibody in puromycin aminonucleoside nephropathy. Kidney Int 51: 301-309, 1997.

17. Sellke FW, Laham RJ, Edelman ER, Pearlman JD and Simons M: Therapeutic angiogenesis with basic fibroblast growth factor: Technique and early results. Ann Thorac Surg 65: 1540-1544, 1998.

18. Wu DQ, Qiu F, Wang T, Jiang XJ, Zhang XZ and Zhuo RX: Toward the development of partially biodegradable and injectable thermoresponsive hydrogels for potential biomedical applications. ACS Appl Mater Interfaces 1: 319-327, 2009. 
19. He YY, Wen Y, Zheng XX and Jiang XJ: Intramyocardia delivery of HMGB1 by a novel thermosensitive hydrogel attenuates cardiac remodeling and improves cardiac function after myocardial infarction. J Cardiovasc Pharmacol 61: 283-290, 2013.

20. Wan WG, Jiang XJ, Li XY, Zhang C, Yi X, Ren S and Zhang XZ: Enhanced cardioprotective effects mediated by plasmid containing the short-hairpin RNA of angiotensin converting enzyme with a biodegradable hydrogel after myocardial infarction. J Biomed Mater Res A 102: 3452-3458, 2014.

21. Simons M,Annex BH,LahamRJ,Kleiman N,HenryT,DauermanH, Udelson JE, Gervino EV, Pike M, Whitehouse MJ, et al: Pharmacological treatment of coronary artery disease with recombinant fibroblast growth factor-2: Double-blind, randomized, controlled clinical trial. Circulation 105: 788-793, 2002.

22. Rajanayagam MA, Shou M, Thirumurti V, Lazarous DF, Quyyumi AA, Goncalves L, Stiber J, Epstein SE and Unger EF: Intracoronary basic fibroblast growth factor enhances myocardial collateral perfusion in dogs. J Am Coll Cardiol 35: 519-526, 2000.

23. Laham RJ, Rezaee M, Post M, Sellke FW, Braeckman RA, Hung D and Simons M: Intracoronary and intravenous administration of basic fibroblast growth factor: Myocardial and tissue distribution. Drug Metab Dispos 27: 821-826, 1999.

24. Lazarous DF, Shou M, Stiber JA, Dadhania DM, Thirumurti V, Hodge E and Unger EF: Pharmacodynamics of basic fibroblast growth factor: Route of administration determines myocardial and systemic distribution. Cardiovasc Res 36: 78-85, 1997.

25. Kornowski R, Fuchs S, Leon MB and Epstein SE: Delivery strategies to achieve therapeutic myocardial angiogenesis Circulation 101: 454-458, 2000.

26. Kawasuji M, Nagamine H, Ikeda M, Sakakibara N, Takemura H, Fujii S and Watanabe Y: Therapeutic angiogenesis with intramyocardial administration of basic fibroblast growth factor. Ann Thorac Surg 69: 1155-1161, 2000.

27. Dai W, Wold LE, Dow JS and Kloner RA: Thickening of the infarcted wall by collagen injection improves left ventricular function in rats: A novel approach to preserve cardiac function after myocardial infarction. J Am Coll Cardiol 46: 714-719, 2005.

28. Christman KL and Lee RJ: Biomaterials for the treatment of myocardial infarction. J Am Coll Cardiol 48: 907-913, 2006.
29. Landa N, Miller L, Feinberg MS, Holbova R, Shachar M, Freeman I, Cohen S and Leor J: Effect of injectable alginate implant on cardiac remodeling and function after recent and old infarcts in rat. Circulation 117: 1388-1396, 2008

30. Davis ME, Motion JP, Narmoneva DA, Takahashi T, Hakuno D, Kamm RD, Zhang S and Lee RT: Injectable self-assembling peptide nanofibers create intramyocardial microenvironments for endothelial cells. Circulation 111: 442-450, 2005

31. Jiang XJ, Wang T, Li XY, Wu DQ, Zheng ZB, Zhang JF, Chen JL, Peng B, Jiang H, Huang C and Zhang XZ: Injection of a novel synthetic hydrogel preserves left ventricle function after myocardial infarction. J Biomed Mater Res A 90: 472-477, 2009.

32. Garbern JC, Minami E, Stayton PS and Murry CE: Delivery of basic fibroblast growth factor with a $\mathrm{pH}$-responsive, injectable hydrogel to improve angiogenesis in infarcted myocardium. Biomaterials 32: 2407-2416, 2011.

33. Lopez JJ, Edelman ER, Stamler A, Hibberd MG, Prasad P, Caputo RP, Carrozza JP, Douglas PS, Sellke FW and Simons M: Basic fibroblast growth factor in a porcine model of chronic myocardial ischemia: A comparison of angiographic, echocardiographic and coronary flow parameters. J Pharmacol Exp Ther 282: 385-390, 1997.

34. Fathi E, Nassiri SM, Atyabi N, Ahmadi SH, Imani M, Farahzadi R, Rabbani S, Akhlaghpour S, Sahebjam M and Taheri M: Induction of angiogenesis via topical delivery of basic-fibroblast growth factor from polyvinyl alcohol-dextran blend hydrogel in an ovine model of acute myocardial infarction. J Tissue Eng Regen Med 7: 697-707, 2013.

35. Wang T, Wu DQ, Jiang XJ, Zhang XZ, Li XY, Zhang JF, Zheng ZB, Zhuo R, Jiang H and Huang C: Novel thermosensitive hydrogel injection inhibits post-infarct ventricle remodelling. Eur J Heart Fail 11: 14-19, 2009.

36. Li XY, Wang T, Jiang XJ, Lin T, Wu DQ, Zhang XZ, Okello E, $\mathrm{Xu} \mathrm{HX}$ and Yuan MJ: Injectable hydrogel helps bone marrow-derived mononuclear cells restore infarcted myocardium. Cardiology 115: 194-199, 2010.

37. Zhu H, Jiang X, Li X, Hu M, Wan W, Wen Y, He Y and Zheng X: Intramyocardial delivery of $\mathrm{VEGF}_{165}$ via a novel biodegradable hydrogel induces angiogenesis and improves cardiac function after rat myocardial infarction. Heart Vessels 31: 963-975, 2016. 